\title{
The Breast Tumor Microenvironment: Could Silicone Breast Implant Elicit Breast Carcinoma?
}

This article was published in the following Dove Press journal:

Breast Cancer: Targets and Therapy

\section{Eduardo Fleury (D) \\ Cristiane Nimir (D) ${ }^{2}$ \\ Gabriel Salum D'Alessandro (D) $^{3}$}

'Service of Radiology, IBCC - Instituto Brasileiro de Controle do Câncer, São Paulo, SP, Brazil; ${ }^{2}$ Service of Pathology, FEMME - Laboratório da Mulher, São Paulo, SP, Brazil; ${ }^{3}$ Service of Plastic Surgery, IBCC - Instituto Brasileiro de Controle do Câncer, São Paulo, SP, Brazil
Correspondence: Eduardo Fleury Service of Radiology, IBCC - Instituto Brasileiro de Controle do Câncer, Rua Maestro Chiaffarelli, 409, São Paulo, SP ZIP 01432-030, Brazil

Email edufleury@hotmail.com
Abstract: Complications related to breast implants have received much attention recently. Breast implant-associated anaplastic large cell lymphoma, silicone-induced granuloma of breast implant capsule, and breast implant illness are the main complications reported in the medical literature. However, the literature contains limited evidence regarding the possibility of silicone implants eliciting breast carcinoma. In this manuscript, we propose a theory in which the immune response to silicone breast implant gel bleeding acts as a triggering point for tumor oncogenesis in breast tissue. This hypothesis is derived from our findings of a case of invasive and undifferentiated medullary carcinoma in a patient with a silicone breast implant. The following concepts have been used to support this theory: 1) silicone bleeding from intact breast implants; 2) metaplasia: an adaptation to injury and precursor to dysplasia and cancer; 3) T-cell dysfunction in cancer immunity; 4) inhibitory cells in the tumor microenvironment (TME); 5) morphogenesis and bauplan; and 6) concepts underlying medullary carcinoma. We propose that the inflammatory process in response to silicone particles in the pericapsular glandular tissue favors the development of cellular mutations in specialized epithelial cells. This reverse morphogenesis could have resulted in breast carcinoma of the medullary type in the present case.

Keywords: breast implant-associated anaplastic large cell lymphoma, BIA-ALCL, breast implant illness, BII, silicone granuloma, breast cancer, breast implant

\section{Plain Language Summary}

Recently, many complications are reported regarding breast implants, such as breast implantassociated anaplastic large cell lymphoma (BIA-ALCL), Silicone-Induced Granuloma of Breast Implant Capsule (SIGBIC), and Breast Implant Illness (BII).

For four years, we have carried out a prospective study at our service, where we investigate patients with breast implants who have clinical complaints and were submitted to the Breast Magnetic Resonance scan.

We described the immune response to silicone and correlated it with imaging findings. We also determined the clinical complications of implants, such as joint pain, itching, hardening of the breasts, inflammatory processes, and skin rash.

Over time, we hypothesized that SIGBIC and BIA-ALCL had the same origin, the bleeding gel. Bleeding gel consists of the extravasation of PDMS molecules, a silicone component, which is extremely toxic to humans. We determined the immune response pathways.

We recently had a case of breast carcinoma involving the implant fibrous capsule. The changes in the breast implant were exuberant, and the complaints and imaging features were much more similar to SIGBIC than a typical breast carcinoma.

Based on the case's extensive diagnostic material, we propose a theory of breast carcinoma induction by the implant, based on our evidence and accumulated knowledge. 


\section{Introduction}

Complications related to breast implants have received much attention recently. About five years ago, breast implant-associated anaplastic large cell lymphoma (BIAALCL) gained attention in the mainstream media as a severe complication of silicone implants. However, at that time, less than 300 cases of BIA-ALCL had been reported in the literature, and the disease was also associated with a low mortality rate, which contributed to the narrative that implants were relatively safe. ${ }^{1-3}$

The intracapsular liquid collection presence without inflammatory signs is the primary imaging finding described in the literature for the BIA-ALCL diagnosis. However, the intracapsular collection finding is not specific for BIA-ALCL and can be found in other pathologies such as inflammatory processes (such as autoimmune diseases) and infectious processes. When the collection development is more than one year after surgery, we can characterize it as a late seroma. In this context, warm seromas could be designated when clinical inflammatory complaints are associated, and cold old seromas when the main associated finding present is breast augmentation without inflammatory signs. ${ }^{4,5}$

During the same period, we started a prospective study that aimed to investigate complications related to breast implants. This research was approved by the institutional ethics committee and registered at Plataforma Brasil. In addition to noting that many patients with breast implants showed common breast magnetic resonance imaging (BMRI) abnormalities, we observed that many patients had similar clinical complaints, such as joint pain, itching, skin rash, and hardening of the breasts. On the basis of these findings, we described a disease entity called silicone-induced granuloma of the breast implant capsule (SIGBIC). ${ }^{4}$ For SIGBIC diagnosis, we adopted three diagnostic criteria: a mass with a high signal in $\mathrm{T} 2$ weighted BMRI sequence, late-contrast enhancement, and black-drop signal. We observed that these imaging findings appeared irrespective of the integrity of the implant surface. ${ }^{5}$ We explained that this disease was triggered by an immune response to silicone bleeding from the prosthesis within the external environment. ${ }^{6}$ We also described the disease's pathophysiology and related the most indolent cases to the presence of extracellular silicone (giant cells) and the most aggressive cases to intracellular silicone (foamy histiocytes). The latter presentation was always associated with the presence of intracapsular fluid collection (seroma). ${ }^{7}$

Even without signs of macroscopic rupture and regardless of their surface texture (smooth or textured), all breast implants deteriorate over time. Silicone bleeding is the result of surface deterioration irrespective of macroscopic surface integrity ${ }^{8}$ (Table 1).

The treatment of SIGBIC consists of en bloc capsulectomy and breast implant explantation. Given the history of immunological reactions, replacement with a new implant is usually not possible. ${ }^{6}$ However, the breast tissue injuries caused by this inflammatory reaction are currently not sufficiently understood.

About two years ago, a vast network of women with breast implants shared their personal experiences regarding breast implants on social media (Facebook, Instagram) and reported common clinical symptoms. They called the disease breast implant illness (BII). Although BII was initially treated as a myth by medical academia, ${ }^{8,9,10}$ the Food and Drug Administration (FDA) has subsequently recognized BII and notified silicone manufacturers that they should alert patients to possible complications. The final guidance, "Breast Implants - Certain Labeling Recommendation to Improve Patient Communication,"

Table I Breast Magnetic Resonance (BMR) and Histology Correlation of Silicone-Induced Granuloma of Breast Implant Capsule (SIGBIC)

\begin{tabular}{|c|c|c|}
\hline & Breast Magnetic Resonance & Histology \\
\hline Extracellular silicone & $\begin{array}{l}\text { - Restricted to intracapsular space } \\
\text { - Mass with hypersignal at T2 weighted sequences } \\
\text { - Black-drop signal } \\
\text { - Late contrast enhancement }\end{array}$ & $\begin{array}{l}\text { - Extracellular and intracellular silicone } \\
\text { - Predominance of extracellular silicone } \\
\text { - Germinal centers of lymphocytes } \\
\text { - Diffuse infiltration by lymphocytes at the fibrous capsule }\end{array}$ \\
\hline Intracellular silicone & $\begin{array}{l}\text { - Same findings from intracapsular } \\
\text { - Pericapsular edema } \\
\text { - Lymph node involvement } \\
\text { - Early contrast enhancement of the extracapsular content } \\
\text { - Intracapsular collection }\end{array}$ & $\begin{array}{l}\text { - Extracellular and intracellular silicone } \\
\text { - Predominance of intracellular silicone } \\
\text { - More severe infiltration by lymphocytes at the fibrous capsule } \\
\text { - Collection with histiocytes and lymphocytes }\end{array}$ \\
\hline
\end{tabular}


which is available on the FDA homepage, provides recommendations concerning the content and format for certain labeling information for saline and silicone gel-filled breast implants, including a "black box warning." According to the FDA, textured implants are more likely to have complications. ${ }^{11}$

Nevertheless, information regarding the oncogenic potential of these implants remains scarce, and the literature contains only a few case reports outlining the association between breast implants and carcinoma. ${ }^{12-14}$ Moreover, the existing evidence for the association between breast implants and epithelial carcinoma is contradictory. ${ }^{14,15}$

To address these issues, the present manuscript based on our experience and research aims to hypothesize a possible oncogenesis pathway underlying tumor development in patients with breast implants.

\section{Index Case Report}

This illustrative case report describes the findings of a patient who underwent an aesthetic procedure for breast augmentation with texturized retroglandular silicone implants two years ago. Informed consent and consent to publish were obtained from the patient. The patient was part of a study protocol approved by the institutional ethical committee (Instituto Brasileiro de Controle do Câncer). Study protocol: Plataforma Brasil CAAE: 77,215,317.0.0000.0072. Six months following the surgery, she presented with a palpable mass in the breast, which was diagnosed as a complex cyst on ultrasound and was aspirated and drained. The cytological diagnosis showed no signs of malignancy. Soon after the procedure, the cyst grew in size again, and one year after the surgery, the patient underwent her first BMRI scan. The scan revealed signs of SIGBIC associated with a complex pericapsular cystic formation, communicating with the intracapsular space (Figure 1). Ultrasonography revealed fibrous septa in the topography of the fibrous capsule dehiscence and echotextural changes of the silicone implant, indicating a chemical reaction in the contents of the implant associated to a complex well-defined solidcystic mass with hypervascularized vegetation in breast parenchyma (Figure 2). Complimentary PET-CT (Figure 3) confirmed the findings. Percutaneous biopsy and drainage of the cyst were performed, and the histological diagnosis indicated an undifferentiated epithelial neoplasia.

The patient underwent an excisional surgical biopsy to facilitate therapeutic management, which was performed along with tumorectomy and partial resection of the
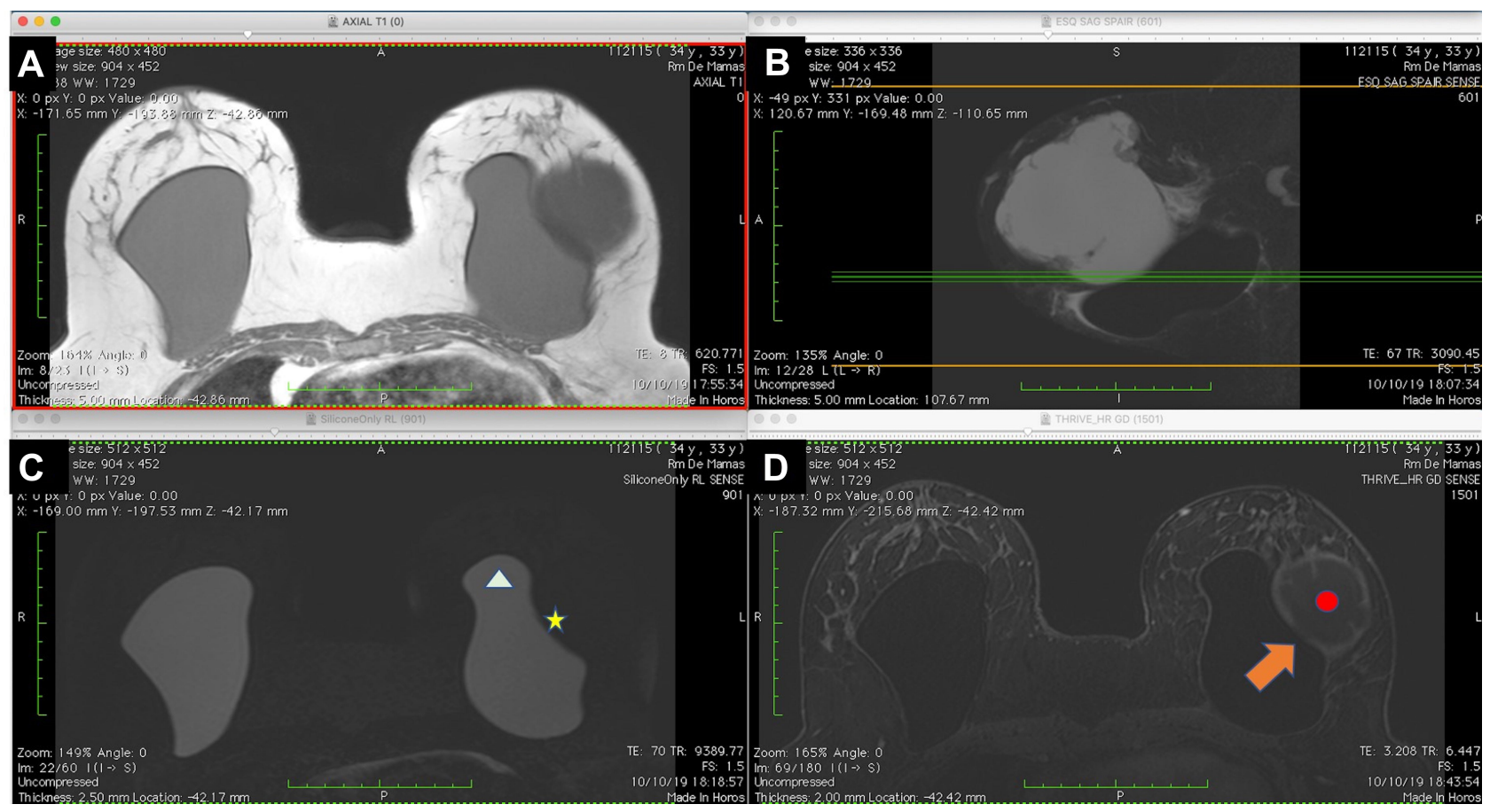

Figure I (A-D). BMRI of the breasts: TI-weighted sequence (A), T2 sequence with fat saturation (B), silicone-sensitive sequence (C), and dynamic phase image (D). The silicone implant (white triangle) is rotated (yellow asterisk). A solid-cystic tumor (red circle) can be observed near the base of the implant (seal), where a discontinued area of the fibrous capsule is present. The discontinued area connects the intracapsular space with the tumor (orange arrow). There is no intracapsular collection. 


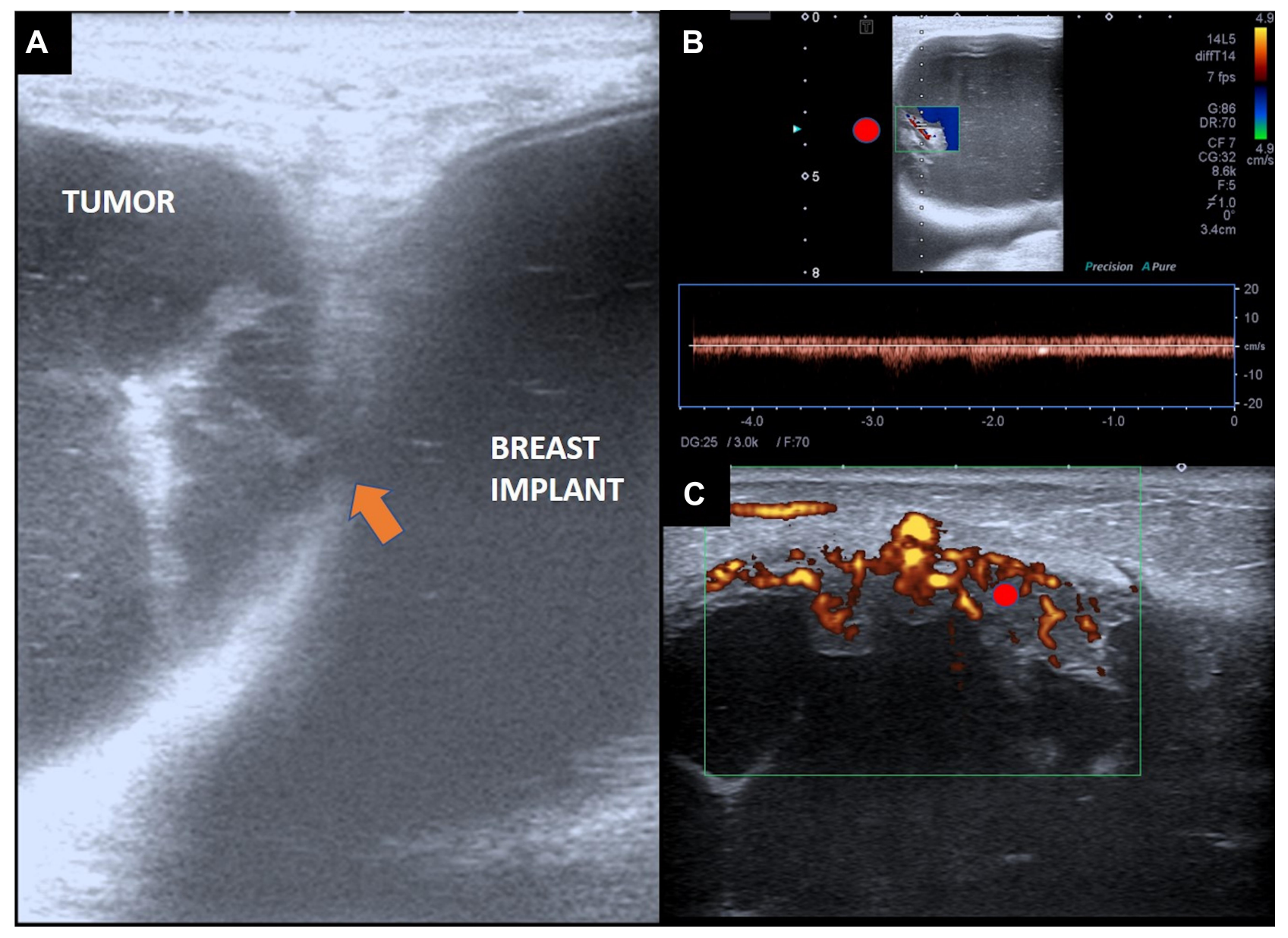

Figure 2 (A-C) B-mode ultrasonography of the tumor (A), with Doppler spectral study (B) and Doppler-flowmeter (C). The silicone implant shows echotextural modification characterized by echogenic lines. An area of discontinuity is visible in the fibrous capsule with interceding septa (orange arrow). The tumor area shows debris indicating high cellularity of the collection (A). The Doppler spectral scan in the mass area of the tumor (red circle) shows a vascular bundle with the arterial flow pattern. The Doppler bean determines the debris motion (blue color in the box) (B). The tumor is well defined by ultrasound scan, with a predominance of vascularization in the parietal mass and no signs of invasion of the adjacent tissue (C).

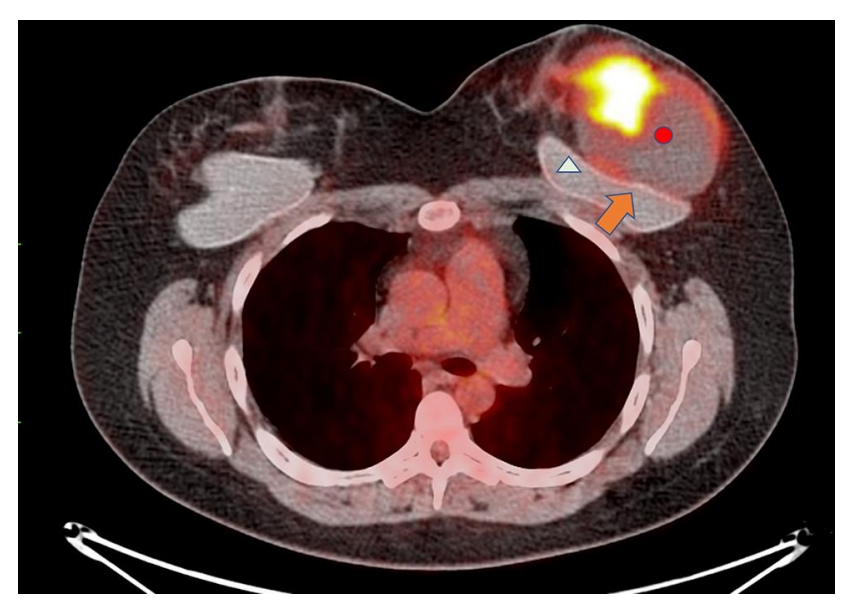

Figure 3 PET-CT of the tumor (red circle) shows ${ }^{18}$ FDG uptake, especially in the mass area inside the tumor. The uptake in the tumor capsule is less intense, but well defined in the acquired images. The silicone implant (white triangle) is rotated, with a low uptake area in the fibrous capsule dehiscence (orange arrow). fibrous capsule (Figure 4). The histological diagnosis was high-grade undifferentiated carcinoma with compromised surgical margins (Figure 5). Treatment with adjuvant chemotherapy and mastectomy was scheduled. Following surgical biopsy and before adjuvant chemotherapy, control BMRI performed for documentation purposes revealed no tumor remnants or lymph node enlargement, two weeks after the surgery. The only change in BMRI, when compared with the first examination, was the silicone implant rotation (Figure 6).

After three months of adjuvant chemotherapy, the patient again underwent BMRI for preoperative staging. BMRI revealed an intracapsular mass formation in the fibrous capsule in the projection of the breast implant's deteriorated area, along with an associated intracapsular 


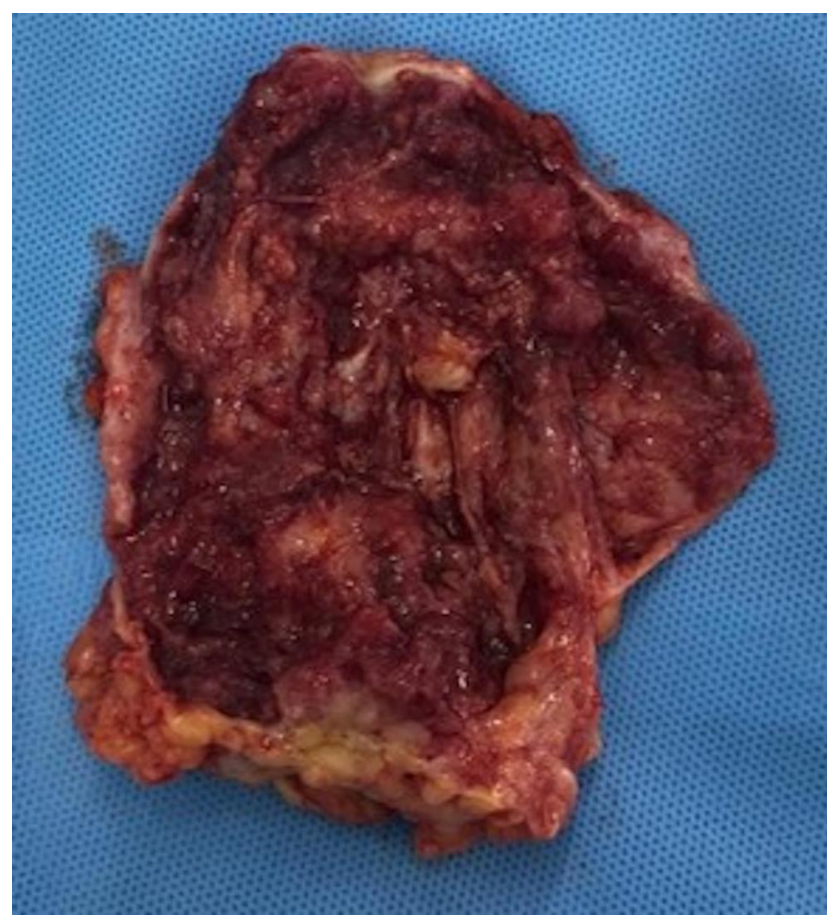

Figure 4 Macroscopic assessment of tumorectomy. Macroscopic assessment of the tumor shows wine vegetation with thick serous-bloody content. The material was friable on palpation and margins were compromised.
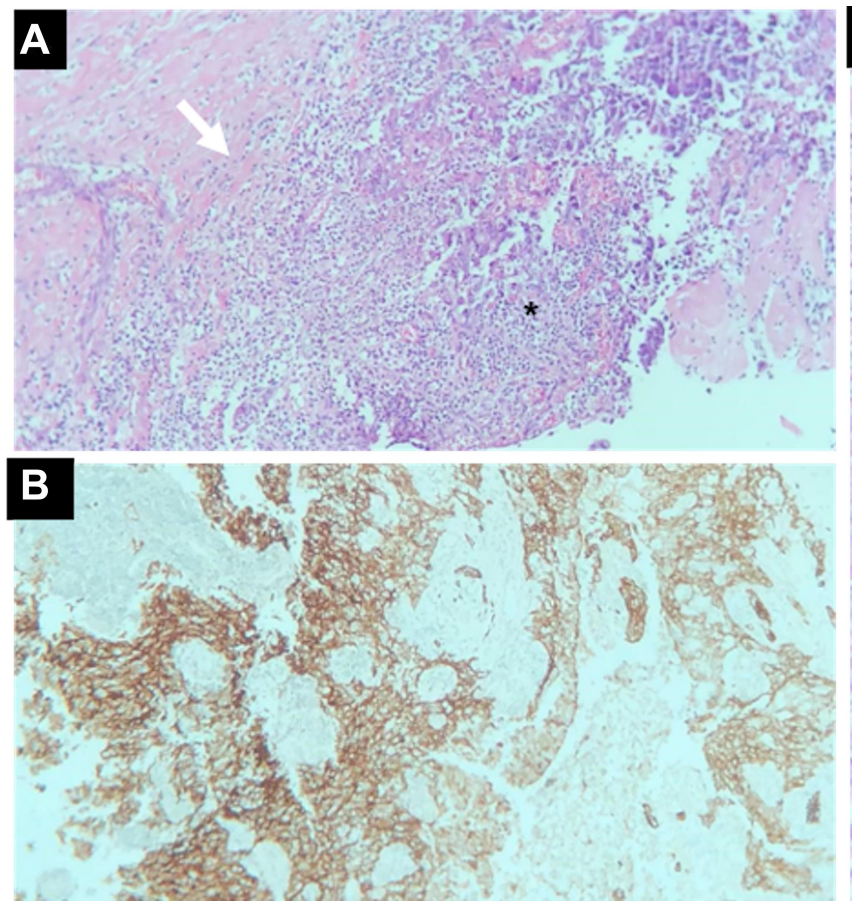

collection. The findings were compatible with those of SIGBIC, inferring silicone gel bleeding (Figure 7). Two weeks later, the macroscopic study of the fibrous capsule after mastectomy confirmed the signs of silicone leakage into the intracapsular space owing to the presence of fibrous septa and thick collection (Figure 8). Histological assessment of the mastectomy product revealed no tumor remnants. The fibrous capsule showed infiltrative areas of the lymphocyte matrix without atypia and with intermingling foamy histiocytes, confirming the diagnosis of SIGBIC. No undifferentiated cells were found (Figure 9). The breast implant showed a microscopic fissure with an internal content exposure area (Figure 10).

\section{Background Metaplasia}

Metaplasia is essentially an adaptation to injury and a precursor to dysplasia and cancer. This process involves the replacement of a mature cell with another, caused by an injury. It can be induced or accelerated by different abnormal stimuli to the tissue

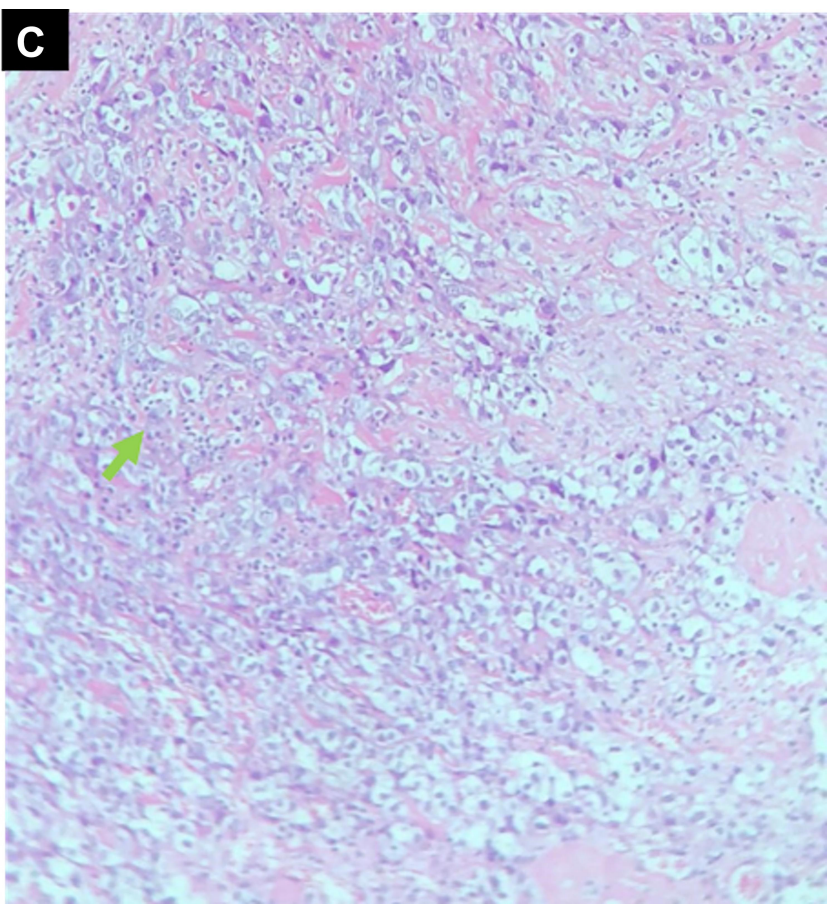

Figure 5 (A-C) Tumor histology with the diagnosis of medullary carcinoma. Microscopy at lower magnification demonstrates undifferentiated cells with syncytial growth, intense inflammatory infiltrate with a predominance of T lymphocytes (black asterisk), and invasion of the capsule (white arrow) (A). The immune profile (pancytokeratins) shows staining the epithelial cells with the tumor markers AEI and AE3 (B). In a greater augmentation, foamy histiocytes are observed between the inflammatory process (green arrow) (C). 

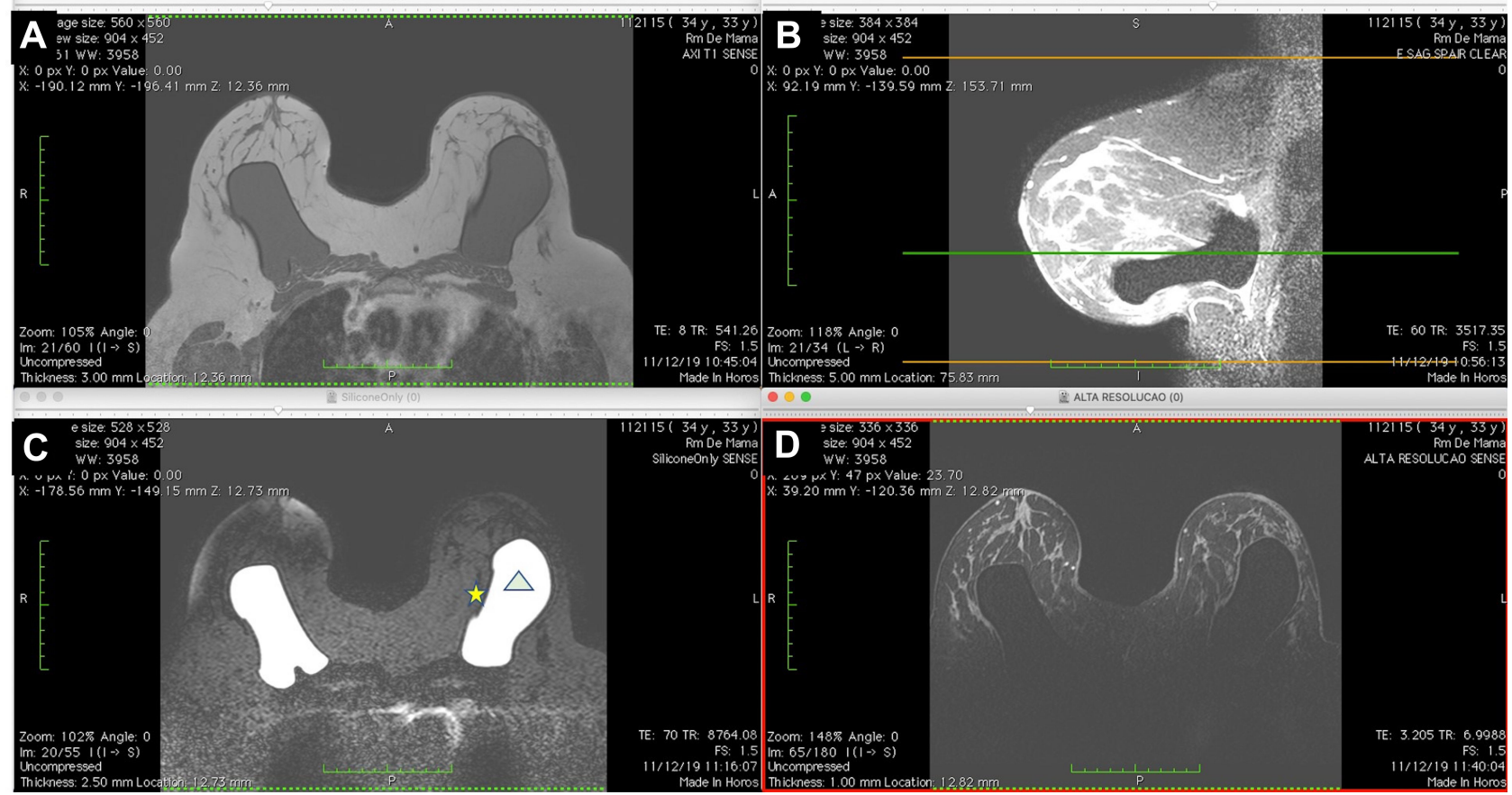

Figure 6 (A-C) Early postoperative BMRI TI-weighted sequence (A), T2 sequence with fat saturation (B), silicone-sensitive sequence (C), and image obtained after contrast administration (D). The silicone implant (white triangle) is rotated to the opposite side to the first exam (yellow asterisk). No residual mass or intracapsular/ extracapsular collections are observed.
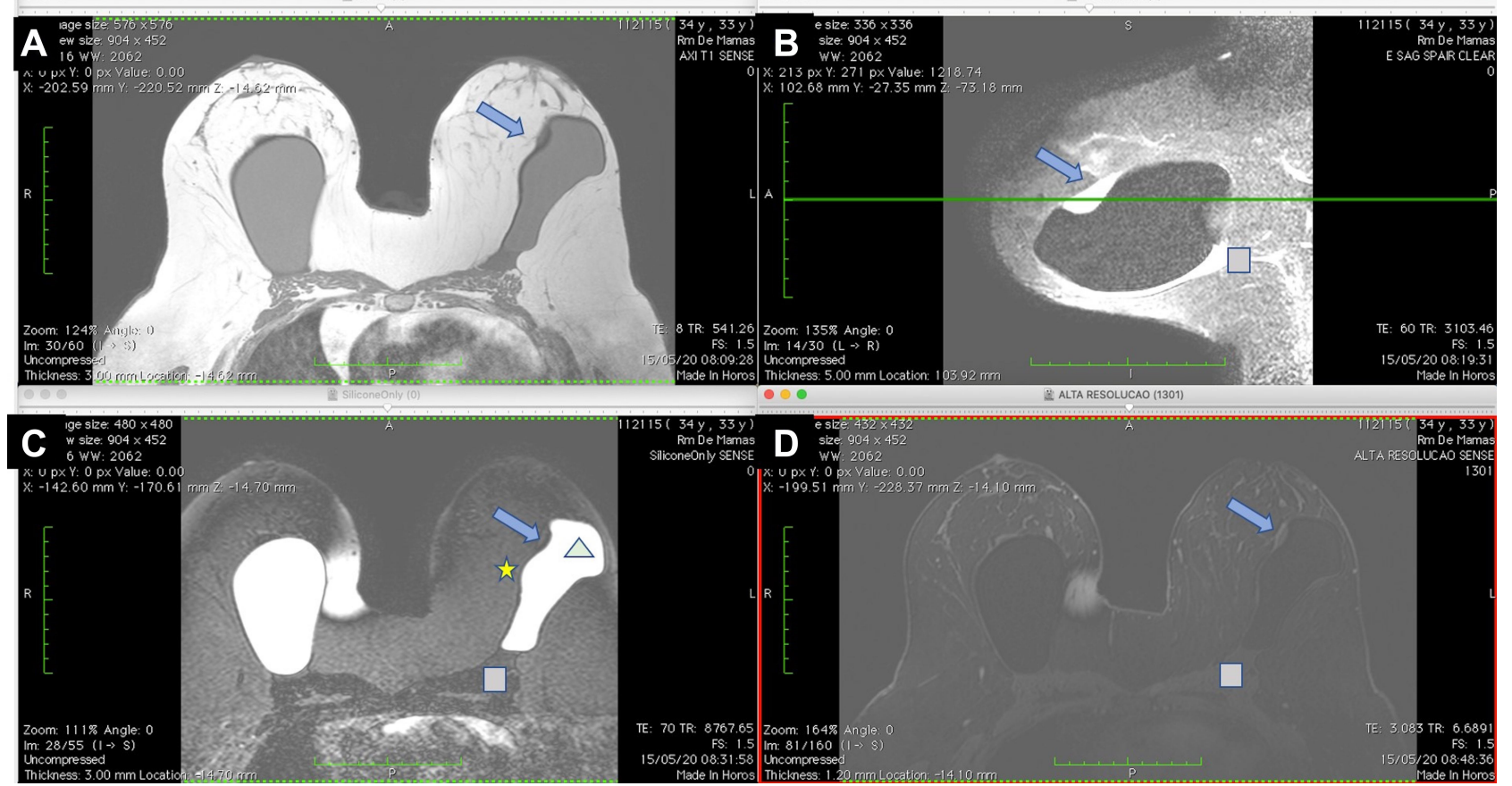

Figure 7 (A-D) BMRI after breast adjuvant chemotherapy showing a TI-weighted sequence (A); T2 sequence with fat saturation (B); silicone-sensitive sequence (C); and image obtained after contrast enhancement (D). The silicone implant (light green triangle) is rotated (yellow asterisk) compared to Figure 9 . However, this figure shows an intracapsular mass formation and late-contrast enhancement in the projection of the implant fractured area (arrow blue) compatible with SIGBIC. A small/intermediary intracapsular collection is observed (gray square). 


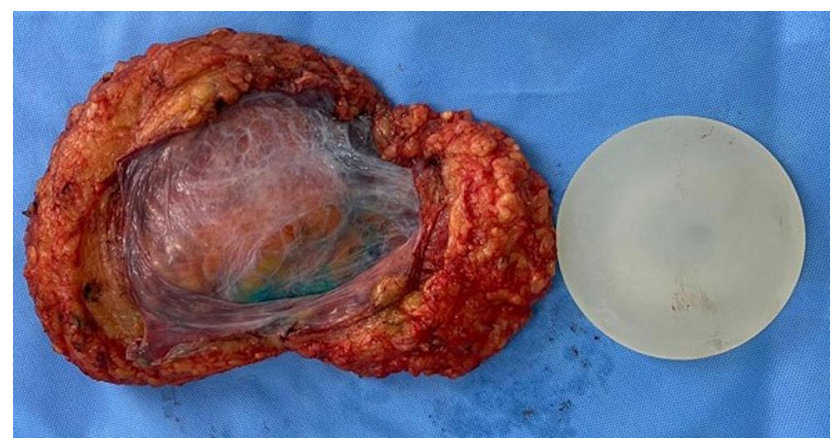

Figure 8 Macroscopic assessment of the mastectomy. Macroscopic assessment of the implant shows the implant surface without visible signs of rupture, with thick fluid in between and fibrous beams. Areas of focal thickening and greater vascularization are visible. microenvironment, such as acid or alkaline stimuli, $\mathrm{pH}$ changes, hormones, cigarette smoking, and alcohol. The innate cells facing this stimulus adapt to the new stress agent, changing their identity. When persistent, metaplasia can progress to dysplasia and malignancy. A classic example of metaplasia is Barrett's mucosa in the esophagus, wherein 1 in 860 individuals $(0.12 \%)$ show transformation of intestinal esophageal metaplasia to cancer $^{16,17}$ (Figure 11).

Malignant transformation of healthy cells occurs through different phases. The first phase is adaptive, in which natural cells are stressed by the new agent. This is

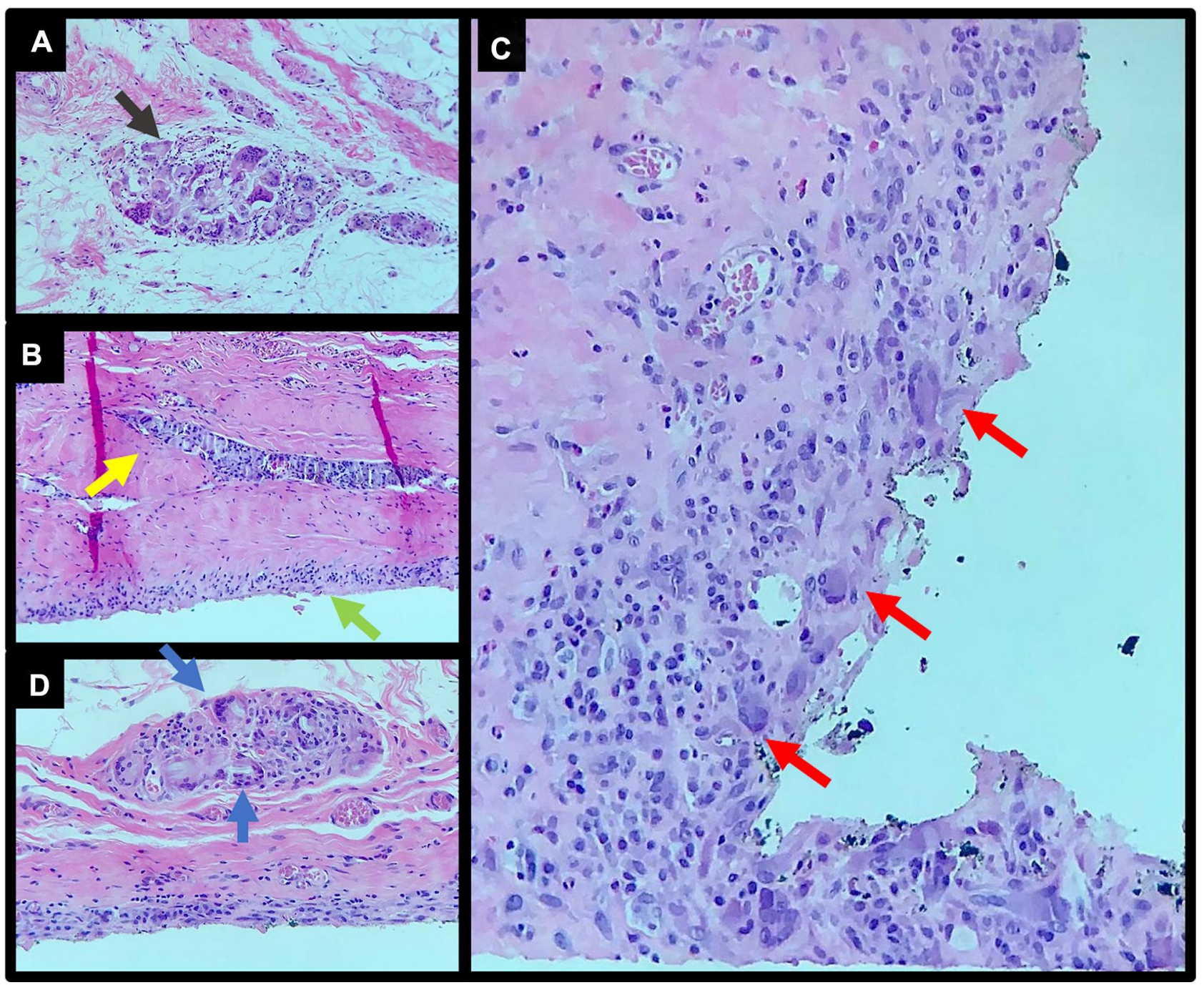

Figure 9 (A-D) Histological assessment of the fibrous capsule after mastectomy. There is no residual tumor on examination. Accumulation of histiocytes between the fibrous capsule (black arrow) (A). Another area with an accumulation of macrophages in the fibrous capsule (green, blue and yellow arrow) with a giant cell reaction (B and D). Giant cells and foamy histiocytes (red arrow) (C). 

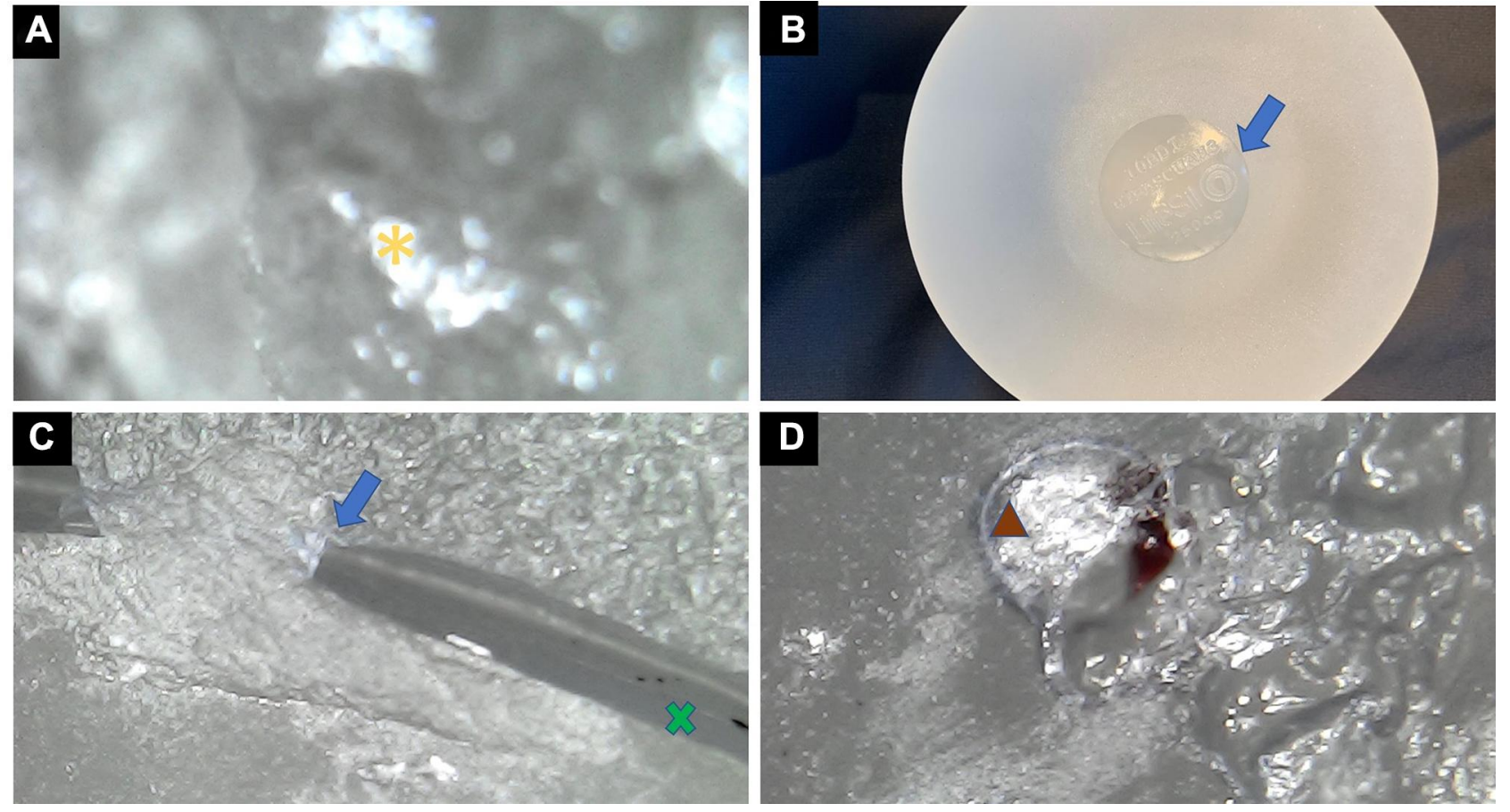

Figure 10 (A-D) Evaluation of the breast implant. Microscopy of the implant's interior with a $1600 \times$ magnification showing signs of chemical reaction of the internal content, with droplets inside the implant (yellow asterisk) (A). Macroscopic assessment shows that the implant is intact (B). The transition surface between the textured and smooth surfaces on the implant seal shows fissured detachment of the surfaces with exposure of the internal content (blue arrow). The interior of the implant also shows changes in color, with a cloudier appearance containing foreign bodies ( $x$ green) $(\mathbf{C})$. At the surface transition, deterioration of the implant surface is visible with hematic material in between (brown triangle) (D).

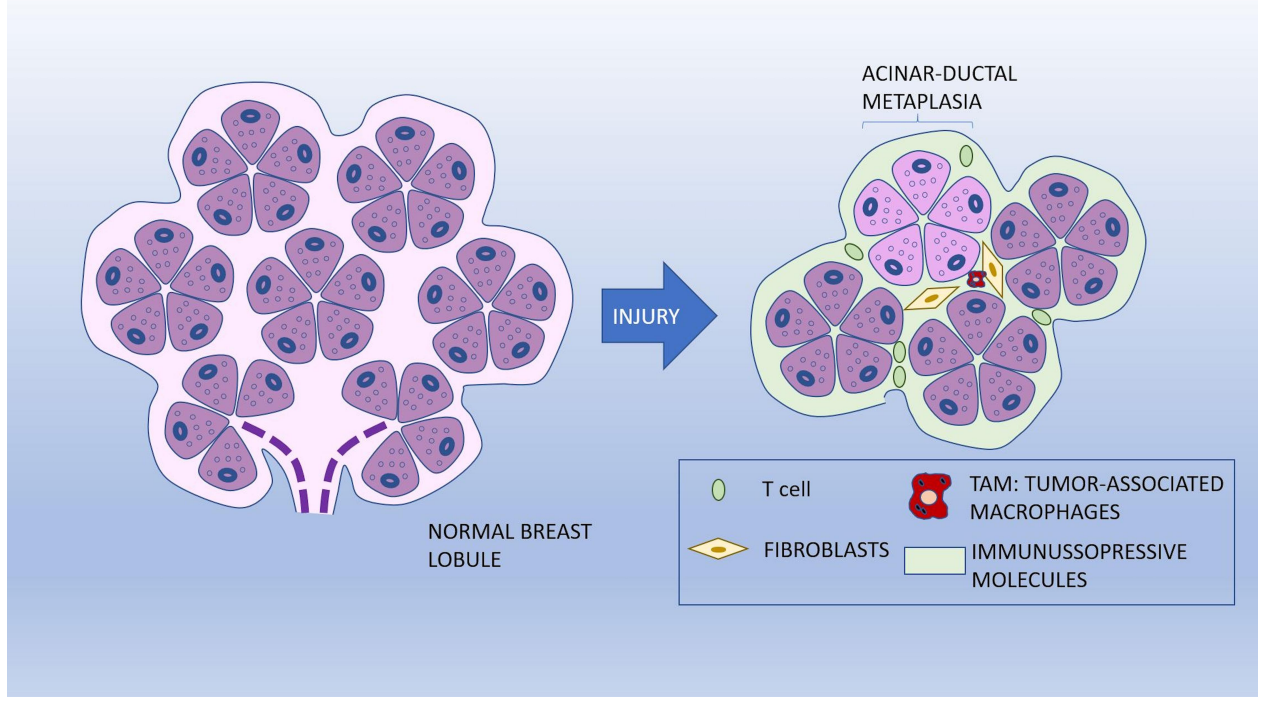

Figure I I Schematic illustration of acinar-ductal metaplasia (ADM).

followed by the oncogenic phase, which involves a transformation from metaplasia to dysplasia. ${ }^{16}$

One type of metaplasia that can be found in the breast is acinar-ductal metaplasia (ADM). ${ }^{18} \mathrm{ADM}$ is also seen in the pancreas and may be related to an acute or chronic inflammatory process. In mice, ADM is a well-defined precursor of pancreatic intraepithelial neoplasia, which may eventually progress to pancreatic ductal adenocarcinoma. Pancreatic metaplasia can be reversed by removing inflammatory stimuli. ${ }^{19,20}$

Metaplasia, caused by non-epithelial factors, may be related to immune cells, inflammatory cells, fibroblasts, 
and the stimulation of external stress agents. These agents force native cells to lose their specific identity and lineage. Pro-inflammatory and immunological cues influence epithelial cell signaling and the induction of metaplasia. Immune cells play an essential role in this process. For example, the inflammatory cytokines secreted by macrophages play important roles in pancreatic ADM, with contributions from macrophage-released matrix metalloproteinases. Similarly, interleukin-3 has been recently shown to change the populations of inflammatory macrophages; from a subpopulation of inflammatory macrophages to a subpopulation of macrophages alternatively activated in ADM. ${ }^{16}$

In the present case, the breast tissue was permanently exposed to silicone bleeding. The implant was a continuous and persistent generator of the antigen that could have caused abnormal stimuli in the tumor microenvironment (TME).

\section{T-Cell Dysfunction in Cancer Immunity}

Overexposure to an antigen can result in T-cell dysfunction. In addition, chronic stimulation by the antigen results in persistent expression of programmed cell death protein by cytoplasmic NFAT $1 .^{21,23,24}$ T-cell dysfunction can be represented by $\mathrm{T}$-cell exhaustion (Tex), anergic T cells, and senescent T cells. Senescent T cells represent the final state of differentiation due to a repeated stimulus, which involves an irreversible cell cycle and shortening of the telomere. ${ }^{18,22}$

Fluid analysis of the index case showed foamy histiocytes that could have elicited T-cell dysfunction.

\section{Inhibitory Cells in the TME}

A variety of cell types participate in the stimulation or inhibition of tumor growth in the TME. Immunosuppressive cells, such as regulatory $\mathrm{T}$ cells (Tregs), tumor-associated macrophages (TAMs), myeloid-derived suppressor cells (MDSCs), cancer-associated fibroblasts and adipocytes, and endothelial cells, are present in the TME and contribute to the dysfunction. $^{24}$

Treg cells, the largest population of $\mathrm{CD}^{+} \mathrm{T}$ cells infiltrating the TME, can inhibit T-cell-mediated antitumor immunity. Treg cells generally interrupt T-cell activation, proliferation, and survival by producing immunosuppressive molecules, including transforming growth factor-beta and interleukin-10 (IL-10). However, therapeutic approaches targeting the antibodies in Treg cells can deplete Treg cells, reverse T-cell dysfunction, and restore
T-cell antitumor immunity and immune surveillance for cancer cells. $^{24-26}$

TAMs suppress T-cell antitumor immunity and promote tumor development, involving functions such as sustainable accumulation of Treg cells, deregulation of vascularization by expression of chemokines and amino acid-degrading enzymes, and promotion of T-cell dysfunction. Likewise, MDSCs enter the TME in an aberrant manner, produce nitric oxide and reactive oxygen species, and express arginase 1 and IDO, promoting T-cell dysfunction. $^{24,27}$

The case described in the present study showed the presence of inflammatory cells such as foamy histiocytes and lymphocytes, which were associated with undifferentiated epithelial cells and silicone corpuscles.

\section{Morphogenesis and Bauplan}

Morphogenesis is a process of "forward" genomic folding that increases the complexity of processing topobiological information, with plasticity loss occurring at its highest levels. $^{28,29}$

The term bauplan was introduced to characterize the morphological features of different species. In the context of tissues, the bauplan in the TME allows cells to perceive topobiological information presented in the vicinity of a morphogenetic field by signaling (ie, epitopes, chemical gradients) and physical phenomena (ie, pressure, tension, bioelectric events) to respond to activities programmed as proliferation, migration, aggregation, differentiation, quiescence, apoptosis, etc. Each bauplan shows two characteristics: 1) complexity of the processed topobiological information and 2) plasticity, allowing the cells to adapt their bauplan to the topological information provided by the morphogenetic fields of the adjacent-cell populations. ${ }^{28,30}$

The mature cells at the top of these layers' hierarchies have minimal to no plasticity but are highly specialized. This last bauplan needs to adjust the continuous loss of cells and work in conjunction with innate and adaptive immune systems to preserve the tissue's integrity. ${ }^{28,31}$

Hockel and $\mathrm{Ben}^{28}$ also postulated that within relevant clinical conditions, a malignant tumor is initiated by (epi-) genetic alterations, in which cell proliferation is increased, and the final layer of the bauplan is disturbed, allowing cell divisions in domains that are normally restricted from differentiation. For epithelial cancer, these manifestations are microscopically observed in dysplasia or carcinoma in situ. Genetic changes determining the function of oncogenes and resulting in the loss of tumor suppressor genes 
are known as driver mutations. This process of tumor development is called reverse morphogenesis. ${ }^{28,32}$

In the case described here, the exposition of the intracapsular inflammatory content to the pericapsular space could have caused injury to the specialized epithelial cells and triggered a local inflammatory response and metaplasia.

\section{Invasive Carcinoma of the Non-Special Type with Medullar Features}

Invasive carcinomas of the non-special type with medullary features, categorized as medullary carcinomas (MCs), represent less than $5 \%$ of breast carcinomas. The diagnosis of MC is specific, with five diagnostic criteria being adopted: complete circumscribed lesion, growth of syncytial pattern in at least $75 \%$ of the tumor, intermediate/high nuclear grade, diffuse lymphocytic infiltrate, and absence of intraductal component or glandular differentiation. Despite the poor histological differentiation and basallike phenotype, this tumor has a favorable prognosis. ${ }^{33}$

In histological assessments, in addition to the five diagnostic criteria, MCs show an increase in activated cytotoxic lymphocytes, with a predominance of $\mathrm{T}$ cells. Studies have shown that these characteristics reflect an active response of the organism to the tumor, which

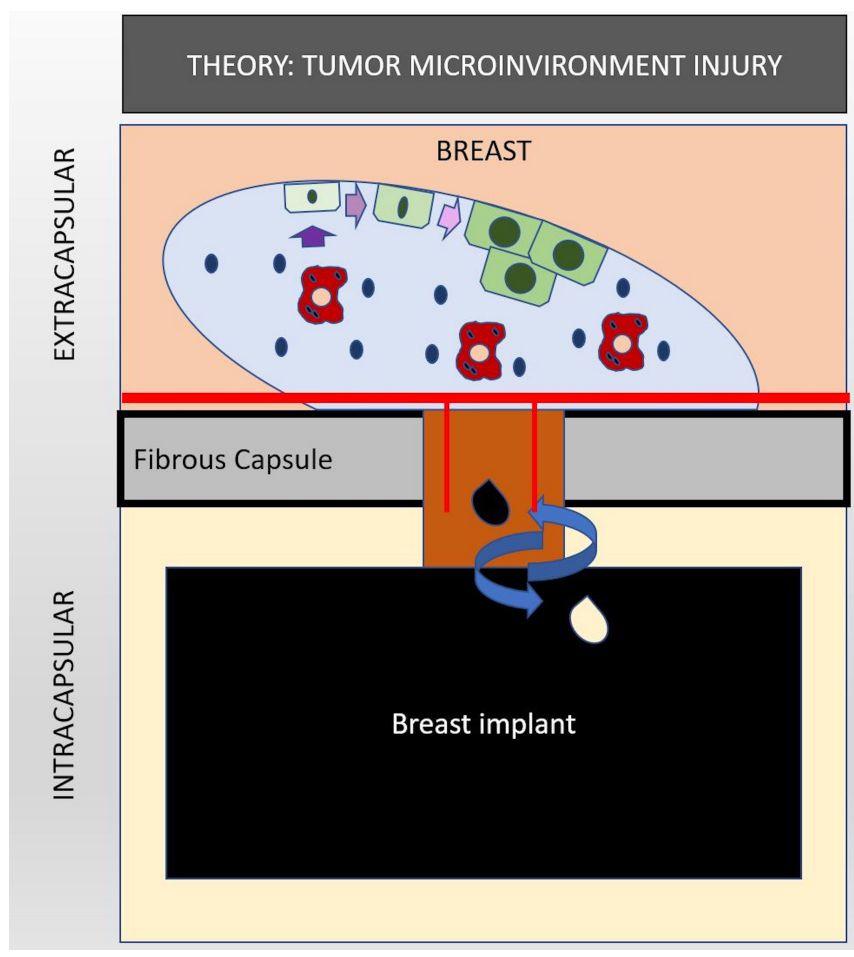

leads to a good prognosis. Mitoses are numerous, and atypical giant cells may be present. ${ }^{33,34}$

\section{Theory}

Our proposed theory is based on the immune response to the bleeding gel of the silicone breast implant serving as a triggering point for tumor oncogenesis in breast tissue. We propose that the inflammatory process in response to silicone particles in the pericapsular glandular tissue favors the development of cellular mutations in specialized epithelial cells. This reverse morphogenesis would result in MC according to the following steps (Figure 12):

Step 1. Breast implant placement with the formation of the fibrous capsule by the host.

Step 2. Deterioration and permeability loss of the implant surface, with exposure and leakage of the silicone into the intracapsular space.

Step 3. Fibrous capsule inflammatory response to polydimethylsiloxane (PDMS4), resulting in intracellular silicone (foamy histiocytes) triggering dysregulation of the inflammatory process with T-cell recruitment and seroma formation.

Step 4. Dehiscence of the fibrous capsule with extrusion of the intracapsular component into the pericapsular space.

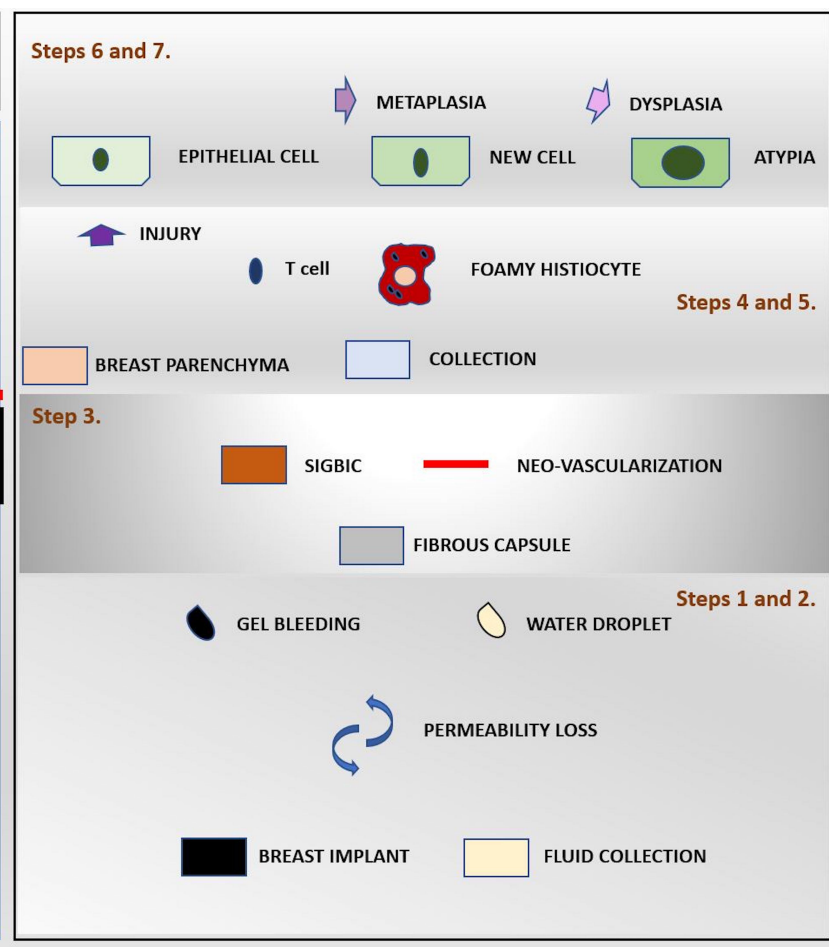

Figure 12 The proposed theory of cell dysplasia induction as a result of silicone bleeding. 
Step 5. Exposure of epithelial cells to the inflammatory process originating from chronic gel bleeding, with predominance of monoclonal $\mathrm{T}$ cells and foamy histiocytes.

Step 6. Changes in the microenvironment of these epithelial cells in the fibrous capsule dehiscence area. Immunosuppression of this microenvironment occurs due to the modulated inflammatory response mediated by $\mathrm{T}$ cells.

Step 7. Dysregulated cell mutation and proliferation of epithelial cells in the presence of mutations.

Step 8. Locoregional invasion of tumor cells, rare.

Step 9. Lymph node metastasis, rare.

\section{Discussion}

Despite the fact that some studies have described the association between breast carcinoma and silicone implants, the pathophysiology of cancer development and the role of silicone particles in the pathway remain unclear.

Cases of BIA-ALCL, BII, and SIGBIC have been reported in the literature. In our prior work, we previously demonstrated the immune response to silicone particle exposure in patients using BMRI scans and the associated histological findings. ${ }^{4-6}$ We have also previously demonstrated that all silicone implants, both smooth and textured, deteriorate over time. ${ }^{8}$ The main result of this deterioration is the gel bleeding or shedding. ${ }^{6}$ The proposed theory describes the possibility of pericapsular breast carcinomas development in patients with breast implants.

To date, the causes of silicone breast implant-related complications remain controversial. There seems to be no consensus in the literature regarding implant safety or possible triggered complications. ${ }^{1-3}$ In 2019, Coroneis et al published an article reporting post-approval FDA studies that assessed long-term implant outcomes in almost 100,000 patients. ${ }^{14}$ Curiously, the article did not point to gel bleeding as a possible cause of implant-related harm and recommended postoperative follow-up of implants by ultrasound screening. Another study from the same year showed an improvement in clinical symptoms and related complications from gel bleeding in patients with BII who underwent an explant procedure. ${ }^{35}$

First, we questioned whether the product of the inflammatory response to the silicone corpuscles could be carcinogenic when in contact with the glandular tissue. Could this content's physical aggression to epithelial cells, in association with the immunosuppression in the TME by $\mathrm{T}$ cells and TAMs, be sufficient to determine the carcinogenesis of the cells? Would the carcinogenesis be a cause or a consequence in such cases? Conceptually, the good prognosis of $\mathrm{MC}$ is attributable to the presence of $\mathrm{T}$ cells, which ensures a good immune response to the tumor and facilitates its treatment. In contrast, a lack of locally aggressive behavior along with well-delimited characteristics of these highly undifferentiated tumors are extremely interesting behaviors. On the basis of these findings, the reverse can be speculated - the reverse morphogenesis of the epithelial cells may be due to the aggression of the differentiated cells in the last bauplan layer, favored by the immunosuppressive effects of $\mathrm{T}$ cells, TAMs, and their products. Therefore, carcinoma may be a consequence of the inflammatory process rather than the inflammatory process resulting from the carcinoma.

After the excisional biopsy, no tumor remnants were observed by BMRI without neoadjuvant treatment, despite the margins compromised by the surgical biopsy (Figure 4). The only evolutive imaging change observed was the implant's rotation compared to the previous study. The shell's discontinuity area was facing the contralateral side of the fibrous capsule. These follow-up BMRI findings for undifferentiated tumors are not trivial in clinical practice. In the following pre-surgical study, intracapsular SIGBIC was observed in the shell's discontinuity projection, confirmed by histology.

The proposed pathological pathway is not exclusive to cancer development. It is worth mentioning that the pathology is usually multifactorial. Thus, the response to the same pathogen can vary among individuals. In this context, in a recent study, Onneking et al described the possible cellular toxicity exerted by silicone. ${ }^{36}$ According to the authors, silicone implants are composed of crosslinked alloys of polydimethylsiloxane chains in threedimensional networks. Since the chains are cross-linked, lower molecular weight silicone particles with linear or cyclic structures, including D4, D5, and D6, filled the empty spaces. In this study, the authors tested the toxicity of free silicone particles in vitro on Jurkat cells (human $\mathrm{T}$ lymphoblast non-adherent lineage). The silicone used for the test was D4 (octadimethylcyclotetrasiloxane) since it has the lowest molecular weight and is the most susceptible to leakage. D4 is hydrophobic, poorly soluble in water, and lipophilic, allowing the particle to pass through the Jurkat membrane. The study also showed that the toxicity of silicone varied depending on the cell type. D4 determined the cell death of Jurkat cells. In HeLa cells (cervical carcinoma epithelial cells), toxicity was less efficient, whereas in HEp-2 cells (human epithelial cells), cell 
death was not observed. ${ }^{36}$ The results presented in the article suggest that in addition to the mechanism proposed by our theory, the direct toxicity of silicone in T cells and eventually on epithelial cells in the TME could catalyze the process of oncogenesis.

A recently published article aimed to determine the association between breast cancer recurrence in patients undergoing mastectomy. The article concluded that textured implants have higher recurrence rates of cancer than smooth implants. However, the authors assumed that the recurrence mechanism could not be clarified by the data collected. The recurrence rate described in that study is the same as that reported in the literature after mastectomy, varying between $5 \%$ and $10 \%$. A bias of the manuscript was the high prevalence of patients with textured implants compared to smooth implants. ${ }^{14}$ A similar debate was observed in relation to tumor recurrence in patients undergoing autologous fat transfer (AFT). For both examples, we believe that tumor recurrence was more related to the type of oncoplastic surgery than to oncogenic factors, such as the implant surface type and AFT. ${ }^{37}$

In early 2020, La Forgia et al described the earlier BIA-ALCL imaging findings, including periprosthetic involvement. ${ }^{38-40}$ The manuscript findings are similar to those found in our SIGBIC articles. We have described two types of silicone in the fibrous capsule: the extracellular silicone (giant cell), a capsule "scar" result of the inflammation process. Furthermore, the intracellular silicone (foamy histiocyte) when we have a macrophagicmediated $\mathrm{T}$ cell activation often associated with an intracapsular fluid collection and fibrin. We divided SIGBIC into three categories: 1) intracapsular SIGBIC, 2) SIGBIC with extracapsular extension, and 3) mixed SIGBIC associated with seroma. ${ }^{7}$ The referenced articles described similar findings that corroborate that SIGBIC and BIA-ALCL could have the same trigger-point: silicone bleeding. The articles also support our theory, where we hypothesized that the D4 could interfere with the cell morphogenesis process in periprosthetic epithelial cells.

In our prospective study on breast implants, we found carcinoma in $5 \%$ of patients (to be published). However, it was not possible to assess the surface integrity of the implant surface in these cases. For this reason, we chose to use in this hypothesis and theory article a case with extensive documentation by imaging methods, histology, clinical manifestations, and microscopic study of the implant surface to support our findings (Figure 13).

Confirmation of our hypothesis would possibly indicate the presence of many other silicone-induced breast tumors in patients with silicone implants, whose etiology has remained undiagnosed due to the lack of scientific evidence in the literature. In this regard, a better understanding of the underlying oncogenic processes will facilitate the development of an appropriate therapeutic proposal to improve efficiency and reduce morbidity in patient care.

\section{Future Perspectives}

We hope that this theory will lead to further studies correlating breast implants with breast carcinomas. For this, it would be necessary to identify SIGBIC findings in

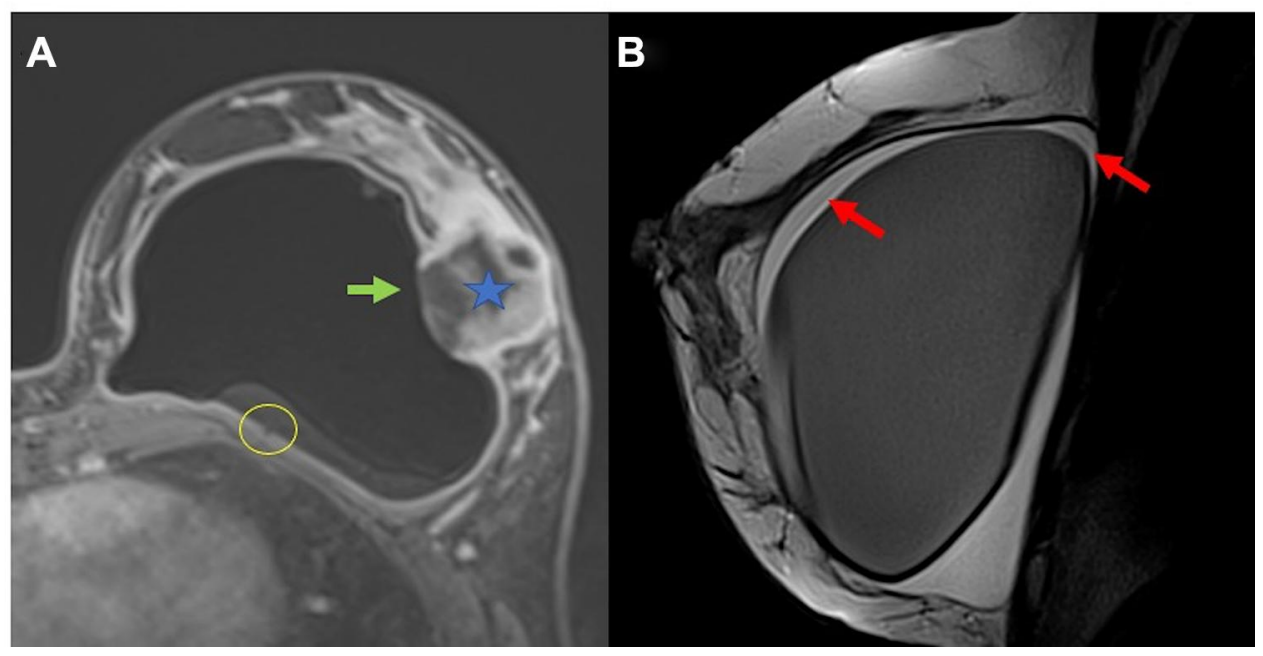

Figure 13 (A and B) Another example of undifferentiated breast carcinoma (blue star). There is a communication between the intracapsular space to the breast tumor. A discontinuity of the fibrous capsule is observed (green arrow). There is also the black-drop signal in fibrous capsule (yellow circle) (A). The red arrow show intracapsular fluid collection with septa inferring fibrin content (B). 
preoperative examinations to investigate the presence of foamy histiocytes on microscopy by pathologists and the microscopic evaluation of the integrity of the silicone implant surface in patients with suspicious lesions in the pericapsular implant space.

Our theory proposed that free silicone could elicit epithelial metaplasia/dysplasia, which could vary in the host response to an external antigen, from more differentiated to undifferentiated tumors. Since this tumor is probably antigen dependent, its treatment can be personalized. Considering these findings, the safety of breast implants must be questioned, given the complexity of the triggered immune response's complications.

\section{Abbreviations}

BIA-ALCL, breast implant-associated anaplastic large cell lymphoma; BMRI, breast magnetic resonance; SIGBIC, silicone-induced granuloma of breast implant capsule; BII, breast implant illness; FDA, Food and Drug Administration; PET-CT, positron-emission tomography; US, ultrasonography; ADM, acinar-ductal metaplasia; PanIN, pancreatic intraepithelial neoplasia; IL, interleukin; STAT3, signal transducers and activators of transcription 3; TNF, tumor necrosis factor; CCL5, CC motif chemokine 5; MMPs, macrophage-released matrix metalloproteinases; SHH, sonic hedgehog; Teff, stroma in the transcription factor ETS2 T cells; TCR, T cell receptor; NFAT, nuclear factor f activated T cells; SPRY2, sprouty homolog 2; PD-1, programmed cell death protein; Tex, T cell exhaustion; TME, tumor microenvironment; Treg cells, regulatory $\mathrm{T}$ cell; TAMs, tumor-associated macrophages; MDSCs, myeloid-derived suppressor cells; TGF$\mathrm{B}$, transforming growth factor-BETA; TIGIT, T cell immunoreceptor with Ig and ITIM domains; MC, medullary carcinoma; ASIA, autoimmune/inflammatory syndrome induced by adjuvants.

\section{Disclosure}

The authors report no conflicts of interest in this work.

\section{References}

1. Jarjis RD, Matzen SH. Anaplastisk storcellet lymfomassocieret til brystimplantater [Breast implant-associated anaplastic large-cell lymphoma]. Ugeskr Laeger. 2015;177(48):V07150625.

2. Kim B, Predmore ZS, Mattke S, van Busum K, Gidengil CA. Breast implant-associated anaplastic large cell lymphoma: updated results from a structured expert consultation process. Plastic and reconstructive surgery. Global Open. 2015;3(1):e296. doi:10.1097/GOX.0000 000000000268
3. Adrada BE, Miranda RN, Rauch GM, et al. Breast implant-associated anaplastic large cell lymphoma: sensitivity, specificity, and findings of imaging studies in 44 patients. Breast Cancer Res Treat. 2014;147 (1):1-14. doi:10.1007/s10549-014-3034-3

4. Fleury EF, Rêgo MM, Ramalho LC, et al. Silicone-induced granuloma of breast implant capsule (SIGBIC): similarities and differences with anaplastic large cell lymphoma (ALCL) and their differential diagnosis. Breast Cancer (Dove Medical Press). 2017;9:133-140. doi:10.2147/BCTT.S126003

5. de Faria Castro Fleury E, Gianini AC, Ayres V, Ramalho LC, Seleti RO, Roveda D. Breast magnetic resonance imaging: tips for the diagnosis of silicone-induced granuloma of a breast implant capsule (SIGBIC). Insights Imaging. 2017;8(4):439-446. doi:10.1007/s13244-017-0564-3

6. Fleury E, Baltzer PAT. Silicone Induced Granuloma of Breast Implant Capsule (SIGBIC) diagnosis: Breast Magnetic Resonance (BMR) sensitivity to detect silicone bleeding. PLoS One. 2020;15(6): e0235050. doi:10.1371/journal.pone.0235050

7. de Faria Castro Fleury E, D'Alessandro GS, Lordelo Wludarski SC. Silicone-Induced Granuloma of Breast Implant Capsule (SIGBIC): histopathology and radiological correlation. $J$ Immunol Res. 2018;2018:6784971. doi:10.1155/2018/6784971

8. de Faria Castro Fleury E. Clear to cloudy II: radiological perspective of silicone breast implants. Eur J Plast Surg. 2020;43:505-508. doi:10.1007/s00238-019-01612-z

9. Rohrich RJ, Kaplan J, Dayan E. Silicone Implant Illness: science versus Myth? Plast Reconstr Surg. 2019;144(1):98-109. doi:10.1097/ PRS.0000000000005710

10. Tang SY, Israel JS, Afifi AM. Breast implant illness: symptoms, patient concerns, and the power of social media. Plast Reconstr Surg. 2017;140:765e-766e. doi:10.1097/PRS.0000000000003785

11. U. S. Food \& Drugs. Fda issues final guidance certain labeling recommendations breast implants; 2020. Available from: https:// www.fda.gov/news-events/press-announcements/fda-issues-final-gui dance-certain-labeling-recommendations-breast-implants. Accessed October 21, 2020.

12. Nakahori R, Takahashi R, Akashi M, et al. Breast carcinoma originating from a silicone granuloma: a case report. World J Surg Oncol. 2015;13:72. doi:10.1186/s12957-015-0509-6

13. Zhou YM, Chaudhry HE, Shah A, Andrews J. Breast squamous cell carcinoma following breast augmentation. Cureus. 2018;10(10): e3405. doi:10.7759/cureus.3405

14. Coroneos CJ, Selber JC, Offodile AC 2nd, Butler CE, Clemens MW. US FDA Breast Implant Postapproval Studies: Long-term Outcomes in 99,993 Patients. Ann Surg. 2019 Jan;269(1):30-36. doi: 10.1097/ SLA.0000000000002990

15. Morgenstern L, Gleischman SH, Michel SL, Rosenberg JE, Knight I, Goodman D. Relation of free silicone to human breast carcinoma. Arch Surg (Chicago, Ill: 1960). 1985;120(5):573-577. doi:10.1001/ archsurg.1985.01390290051008

16. Giroux V, Rustgi AK. Metaplasia: tissue injury adaptation and a precursor to the dysplasia-cancer sequence. Nat Rev Cancer. 2017;17(10):594-604. doi:10.1038/nrc.2017.68

17. Hvid-Jensen F, Pedersen L, Drewes AM, Sørensen HT, Funch-Jensen P. Incidence of adenocarcinoma among patients with Barrett's esophagus. $N$ Engl J Med. 2011;365(15):1375-1383. doi:10.1056/ NEJMoa1103042

18. Ying H, Dey P, Yao W, et al. Genetics and biology of pancreatic ductal adenocarcinoma. Genes Dev. 2016;30(4):355-385. doi:10.11 01/gad.275776.115

19. Behranwala KA, Nasiri N, Abdullah N, Trott PA, Gui GP. Squamous cell carcinoma of the breast: clinico-pathologic implications and outcome. Eur J Surg Oncol. 2003;29(4):386-389. doi:10.1053/ejso.2002.1422

20. Hosoda W, Wood LD. Molecular genetics of pancreatic neoplasms. Surg Pathol Clin. 2016;9(4):685-703. doi:10.1016/j.path.2016.05.011

21. Leone RD, Powell JD. Metabolism of immune cells in cancer. Nat Rev Cancer. 2020. doi:10.1038/s41568-020-0273-y 
22. Ramello MC, Tosello Boari J, Canale FP, et al. Tumor-induced senescent $\mathrm{T}$ cells promote the secretion of pro-inflammatory cytokines and angiogenic factors by human monocytes/macrophages through a mechanism that involves Tim-3 and CD40L. Cell Death Dis. 2014;5(11):e1507. doi:10.1038/cddis.2014.451

23. Zhao Y, Shao Q, Peng G. Exhaustion and senescence: two crucial dysfunctional states of $\mathrm{T}$ cells in the tumor microenvironment. Cell Mol Immunol. 2020;17(1):27-35. doi:10.1038/s41423-019-0344-8

24. Xia A, Zhang Y, Xu J, Yin T, Lu XJ. T cell dysfunction in cancer immunity and immunotherapy. Front Immunol. 2019;10:1719. doi:10.3389/fimmu.2019.01719

25. Bulliard Y, Jolicoeur R, Zhang J, Dranoff G, Wilson NS, Brogdon JL. OX40 engagement depletes intratumoral Tregs via activating Fc $\gamma$ Rs, leading to antitumor efficacy. Immunol Cell Biol. 2014;92(6):475480. doi:10.1038/icb.2014.26

26. Sugiyama D, Nishikawa H, Maeda Y, et al. Anti-CCR4 mAb selectively depletes effector-type FoxP3+CD4+ regulatory T cells, evoking antitumor immune responses in humans. Proc Natl Acad Sci U S A. 2013;110(44):17945-17950. doi:10.1073/pnas.1316796110

27. Colegio OR, Chu NQ, Szabo AL, et al. Functional polarization of tumour-associated macrophages by tumour-derived lactic acid. Nature. 2014;513(7519):559-563. doi:10.1038/nature13490

28. Höckel M, Behn U. The order of cancer: a theory of malignant progression by inverse morphogenesis. Front Oncol. 2019;9:416. doi:10.3389/fonc.2019.00416

29. Razin SV, Ulianov SV. Gene functioning and storage within a folded genome. Cell Mol Biol Lett. 2017;22:18. doi:10.1186/s11658-0170050-4

30. Pfeffer PL. Building principles for constructing a mammalian blastocyst embryo. Biology. 2018;7(3):41. doi:10.3390/biology7030041

31. Engler AJ, Humbert PO, Wehrle-Haller B, Weaver VM. Multiscale modeling of form and function. Science (New York, N Y). 2009;324 (5924):208-212. doi:10.1126/science.1170107
32. Vogelstein B, Papadopoulos N, Velculescu VE, Zhou S, Diaz LA, Kinzler KW. Cancer genome landscapes. Science (New York, $N$ Y). 2013;339(6127):1546-1558. doi:10.1126/science.1235122

33. Limaiem F, Mlika M. Medullary Breast Carcinoma. [Updated 2020 Oct 16]. In: StatPearls [Internet]. Treasure Island (FL): StatPearls Publishing; 2020. Available from: https://www.ncbi.nlm.nih.gov/ books/NBK542292/.

34. Chu Z, Lin H, Liang X, et al. Clinicopathologic characteristics of typical medullary breast carcinoma: a retrospective study of 117 cases. PLoS One. 2014;9(11):e111493. doi:10.1371/journal.pone.01 11493

35. Kappel RM, Pruijn GJM. Explantation of silicone breast implants ameliorates gel bleed related health complaints in women with breast implant illness. Clin Med Rev Case Rep. 2020;7:301. doi:10.23937/ 2378-3656/1410301

36. Onnekink C, Kappel RM, Boelens WC, Pruijn G. Low molecular weight silicones induce cell death in cultured cells. Sci Rep. 2020;10 (1):9558. doi:10.1038/s41598-020-66666-7

37. Sorrentino L, Regolo L, Scoccia E, et al. Autologous fat transfer after breast cancer surgery: an exact-matching study on the long-term oncological safety. Eur J Surg Oncol. 2019;45(10):1827-1834. doi:10.1016/j.ejso.2019.05.013

38. La Forgia D, Moschetta M, Fausto A, et al. Anaplastic large-cell periprosthetic lymphoma of the breast: could fibrin be an early radiological indicator of the presence of disease? J BUON. 2019;24 (5):1889-1897.

39. La Forgia D, Moschetta M, Fausto A, et al. Early indicators in anaplastic large-cell periprosthetic lymphoma of the breast: clarifications. J BUON. 2020;25(4):2127-2128.

40. La Forgia D, Catino A, Fausto A, et al. Diagnostic challenges and potential early indicators of breast periprosthetic anaplastic large cell lymphoma: a case report. Medicine. 2020;99(30):e21095. doi:10.10 97/MD.0000000000021095
Breast Cancer: Targets and Therapy

\section{Publish your work in this journal}

Breast Cancer - Targets and Therapy is an international, peer-reviewed open access journal focusing on breast cancer research, identification of therapeutic targets and the optimal use of preventative and integrated treatment interventions to achieve improved outcomes, enhanced survival and quality of life for the cancer patient.

\section{Dovepress}

The manuscript management system is completely online and includes a very quick and fair peer-review system, which is all easy to use. Visit http://www.dovepress.com/testimonials.php to read real quotes from published authors. 